ESAIM: PROCEEDINGS, July 2007, Vol.18, 57-69

Jean-Frédéric Gerbeau \& Stéphane Labbé, Editors

\title{
A POSTERIORI ERROR ESTIMATES FOR FINITE VOLUME AND MIXED FINITE ELEMENT DISCRETIZATIONS OF CONVECTION-DIFFUSION-REACTION EQUATIONS *,**
}

\author{
MARTIN VOHRALÍK ${ }^{1}$
}

\begin{abstract}
We present in this paper a unified framework for a posteriori error estimation in the finite volume and lowest-order Raviart-Thomas mixed finite element methods. We consider convectiondiffusion-reaction equations on simplicial meshes in two or three space dimensions and pay a particular attention to inhomogeneous and anisotropic diffusion-dispersion tensors and to convection dominance, in which case upwind-weighted schemes are considered. Our estimates are derived in the energy (semi-)norm for a locally postprocessed approximate solution preserving the finite volume/mixed finite element fluxes and are of residual type. They give a global upper bound on the error and are fully computable in the sense that all occurring constants are evaluated explicitly, so that they can serve both as indicators for adaptive refinement or for the actual control of the error. Local efficiency and semi-robustness in the sense that the local efficiency constant only depends on local variations in the inhomogeneities and anisotropies and becomes optimal as the local Péclet number gets sufficiently small can also be shown. Moreover, passing from their local to global computation, our estimates become asymptotically exact and asymptotically fully robust with respect to inhomogeneities and anisotropies. We finally present numerical experiments confirming their accuracy and briefly compare the results for the two methods.
\end{abstract}

Résumé. Nous présentons dans ce papier un cadre unifié pour des estimations a posteriori dans les méthodes des volumes finis et des éléments finis mixtes de Raviart-Thomas de plus bas degré. Nous considérons des équations de convection-diffusion-réaction sur de maillages de triangles ou de tétraèdres et nous nous concentrons en particulier sur des tenseurs de diffusion-dispersion inhomogènes et anisotropes et sur des problèmes dominés par la convection. Dans ce dernier cas, la pondération amont est supposée. Nos estimations sont de type résiduel, obtenues dans la (semi-)norme de l'énergie pour une approximation post-traitée localement et conservant les flux de la méthode des volumes finis ou celle des éléments finis mixtes, et elles représentent une borne supérieure globale de l'erreur. Nous avons évalué précisément toutes les constantes figurant dans nos estimations de telle sorte qu'elles soient utilisables non seulement comme des indicateurs de raffinement, mais aussi pour un véritable contrôle de l'erreur dans la solution numérique. On peut aussi montrer l'efficacité locale semi-robuste dans le sens que la constante d'efficacité locale ne dépend que des variations locales dans les inhomogénéités et anisotropies et devient optimale lorsque le nombre de Péclet local devient petit. De plus, passant de leur évaluation locale à leur évaluation globale, nos estimations sont asymptotiquement exactes et, visà-vis des inhomogénéités et des anisotropies, asymptotiquement robustes. Finalement, nous validons la précision de nos estimations par des essais numériques et comparons brièvement les deux méthodes considérées.

\footnotetext{
* The main part of this work was carried out during the author's post-doc stay at Laboratoire de Mathématiques, Université de Paris-Sud and CNRS, Orsay, France

** This work was supported by the GdR MoMaS project "Numerical Simulations and Mathematical Modeling of Underground Nuclear Waste Disposal", PACEN/CNRS, ANDRA, BRGM, CEA, EdF, IRSN, France.

${ }^{1}$ Laboratoire Jacques-Louis Lions, Université Pierre et Marie Curie (Paris 6), B.C. 187, 4 place Jussieu, 75252 Paris, France
}

(C) EDP Sciences, SMAI 2007 


\section{INTRODUCTION}

We consider in this paper the convection-diffusion-reaction problem

$$
\begin{aligned}
& -\nabla \cdot(\mathbf{S} \nabla p)+\nabla \cdot(p \mathbf{w})+r p=f \quad \text { in } \Omega, \\
& p=g \text { on } \Gamma_{\mathrm{D}}, \\
& -\mathbf{S} \nabla p \cdot \mathbf{n}=u \text { on } \Gamma_{\mathrm{N}},
\end{aligned}
$$

where $\mathbf{S}$ is (an inhomogeneous and anisotropic) diffusion-dispersion tensor, $\mathbf{w}$ is a (dominating with respect to $\mathbf{S})$ velocity field, $r$ is a reaction function, $f$ is a source term, and $g$ and $u$ prescribe the Dirichlet and Neumann boundary conditions, respectively. We suppose that $\Omega \subset \mathbb{R}^{d}, d=2,3$, is a polygonal (polyhedral) domain, that $\Gamma_{\mathrm{D}} \cap \Gamma_{\mathrm{N}}=\emptyset, \Gamma_{\mathrm{D}} \cup \Gamma_{\mathrm{N}}=\Gamma:=\partial \Omega$, that $\left|\Gamma_{\mathrm{D}}\right| \neq 0$, where $\left|\Gamma_{\mathrm{D}}\right|$ is the measure of the set $\Gamma_{\mathrm{D}}$, and that $\Gamma_{\text {in }}:=\{\mathbf{x} \in \partial \Omega ; \mathbf{w} \cdot \mathbf{n}<0\} \subset \Gamma_{\mathrm{D}}$. Finally, $\mathbf{n}$ stands for the unit normal vector of $\partial \Omega$, outward to $\Omega$.

A posteriori error estimates are nowadays well established for discretizations of the pure diffusion form of (1.1a)-(1.1c) by the finite element, finite volume, and mixed finite element methods, cf. respectively e.g. Verfürth [19], Nicaise [13], and Carstensen [6]. In most cases, however, the analysis is only given for $\mathbf{S}$ being an identity matrix; an in-depth analysis for the general inhomogeneous (and anisotropic) diffusion tensor was presented by Bernardi and Verfürth [5] or by Petzoldt [17] in the framework of the finite element method. In recent years a posteriori error estimates have been extended to convection-diffusion problems as well. We cite in particular Verfürth [20] who derived reliable, locally efficient, and semi-robust estimates in the energy norm for the finite element method and its stabilized SUPG version. However, only a few works have addressed this issue for the finite volume method - we cite in particular Ohlberger [15] and Nicaise [14] — and, to our knowledge, no results are known up to now for the mixed finite element one.

The purpose of this paper is to present a unified framework for a posteriori error estimation in the finite volume and mixed finite element discretizations of the problem (1.1a)-(1.1c). The basis of our approach is to exploit the particular feature of these methods, which is the conservativity of the discrete fluxes across the sides (edges if $d=2$, faces if $d=3$ ) of the mesh. We first build in Section 3, after collecting notation, assumptions, and details on the continuous problem in Section 2, a postprocessed approximate solution $\tilde{p}_{h}$ which preserves exactly these fluxes and whose point or mean value is in each cell fixed by the original constant approximation. By such a construction, the diffusion-dispersion tensor times the negative of the gradient of $\tilde{p}_{h}$ lies in the $\mathbf{H}(\operatorname{div}, \Omega)$ space. This crucial advantage is however compensated by the fact that $\tilde{p}_{h}$ is nonconforming in the sense that $\tilde{p}_{h} \notin \mathrm{H}^{1}(\Omega)$. The Oswald interpolation operator is then employed to construct a conforming interpolate, which will be used in the a posteriori analysis.

We then in Section 4 present our a posteriori error estimates as well as a sketch of their proof. The derived estimators are associated with the mesh cells, are easily and locally computable, and they are also fully computable in the sense that all occurring constants are evaluated explicitly. They are valid for any cell-centered finite volume scheme, cf. Eymard et al. [10] and [1,7,12], and for the lowest-order Raviart-Thomas mixed finite element scheme and its upwind variant, cf. $[9,18]$ and [8], respectively.

We finally give in Section 5 several remarks and we show there in particular that in the pure diffusion case, passing from their local to global computation, our estimates become globally asymptotically exact and globally asymptotically fully robust with respect to inhomogeneities and anisotropies, i.e. that the ratio of the estimated and actual error goes to one, and this independently of inhomogeneities and anisotropies. Almost optimal efficiency is observed in numerical experiments in Section 6 also in the convection-diffusion-reaction case. Complete proofs of the presented results, several extensions and namely a slightly different form of the estimator in the mixed finite element case, using a modification of the Oswald interpolation operator, detailed discussions, and further numerical experiments are presented in [24,23].

\section{Preliminaries}

We start in this section by some preliminaries. 


\subsection{Partitions of the domain}

We suppose that for all $h>0, \mathcal{T}_{h}$ consists of closed simplices such that $\bar{\Omega}=\bigcup_{K \in \mathcal{T}_{h}} K$ and such that if $K, L \in \mathcal{T}_{h}, K \neq L$, then $K \cap L$ is either an empty set or a common face, edge, or vertex of $K$ and $L$. Let $h_{K}$ denote the diameter of $K$ and let $h:=\max _{K \in \mathcal{T}_{h}} h_{K}$. We make the following shape regularity assumption on the family of triangulations $\left\{\mathcal{T}_{h}\right\}_{h}$, denoting $\kappa_{K}:=|K| / h_{K}^{d}$ :

Assumption (A) (Shape regularity of the meshes)

There exists a constant $\kappa_{\mathcal{T}}>0$ such that $\min _{K \in \mathcal{T}_{h}} \kappa_{K} \geq \kappa_{\mathcal{T}}$ for all $h>0$.

We next denote by $\mathcal{E}_{h}$ the set of all sides of $\mathcal{T}_{h}$, by $\mathcal{E}_{h}^{\text {int }}$ the set of interior sides, by $\mathcal{E}_{h}^{\text {ext }}$ the set of exterior sides, and by $\mathcal{E}_{K}$ the set of all the sides of an element $K \in \mathcal{T}_{h}$. Finally, $\mathcal{E}_{h}^{\mathrm{N}}$ denotes the sides contained in $\overline{\Gamma_{\mathrm{N}}}$ and $\mathcal{E}_{h}^{\mathrm{D}}$ those contained in $\overline{\Gamma_{\mathrm{D}}}$ and $h_{\sigma}$ stands for the diameter of $\sigma \in \mathcal{E}_{h}$.

\subsection{Poincaré, Friedrichs, and trace inequalities}

Let $K$ be a simplex, $\sigma$ its side, and $\varphi \in \mathrm{H}^{1}(K)$. Three inequalities play an essential role in the derivation of our a posteriori error estimates. First, the Poincaré inequality states that

$$
\left\|\varphi-\varphi_{K}\right\|_{K}^{2} \leq C_{\mathrm{P}} h_{K}^{2}\|\nabla \varphi\|_{K}^{2}
$$

where $\varphi_{K}:=(\varphi, 1)_{K} /|K|$ and where the constant $C_{\mathrm{P}}$ can be evaluated as $1 / \pi^{2}$, cf. [16,4]. Second, the following generalized Friedrichs inequality has been proved in [21, Lemma 4.1]:

$$
\left\|\varphi-\varphi_{\sigma}\right\|_{K}^{2} \leq C_{\mathrm{F}, d} h_{K}^{2}\|\nabla \varphi\|_{K}^{2}
$$

where $\varphi_{\sigma}:=\langle\varphi, 1\rangle_{\sigma} /|\sigma|$ and where $C_{\mathrm{F}, d}=3 d$. Finally, the trace inequality states that

$$
\left\|\varphi-\varphi_{\sigma}\right\|_{\sigma}^{2} \leq C_{\mathrm{t}, K, \sigma} h_{K}\|\nabla \varphi\|_{K}^{2} .
$$

It has been shown in [13, Lemma 3.5] that $C_{\mathrm{t}, K, \sigma}=C_{\mathrm{t}, d} h_{K} / h_{\sigma}$, where $C_{\mathrm{t}, d} \approx 1.55416$ if $d=2$ and $C_{\mathrm{t}, d} \approx$ 11.53557 if $d=3$.

\subsection{Assumptions on the data}

We suppose that there exists a basic partition $\widetilde{\mathcal{T}}_{h}$ of $\Omega$ such that the data of the problem (1.1a)-(1.1c) are related to $\widetilde{\mathcal{T}}_{h}$ in the following way:

Assumption (B) (Data)

(B1) $\mathbf{S}_{K}:=\left.\mathbf{S}\right|_{K}$ is a constant, symmetric, bounded, and uniformly positive definite tensor such that $c_{\mathbf{S}, K} \mathbf{v}$. $\mathbf{v} \leq \mathbf{S}_{K} \mathbf{v} \cdot \mathbf{v} \leq C_{\mathbf{S}, K} \mathbf{v} \cdot \mathbf{v}, c_{\mathbf{S}, K}>0, C_{\mathbf{S}, K}>0$, for all $\mathbf{v} \in \mathbb{R}^{d}$ and all $K \in \widetilde{\mathcal{T}}_{h}$

(B2) $\mathbf{w} \in \mathbf{R T}\left(\widetilde{\mathcal{T}}_{h}\right)$ satisfies $|\mathbf{w}|_{K} \mid \leq C_{\mathbf{w}, K}, C_{\mathbf{w}, K} \geq 0$, for all $K \in \widetilde{\mathcal{T}}_{h}$;

(B3) $r$ is a polynomial of degree at most $k$ on each $K \in \widetilde{\mathcal{T}}_{h}$;

(B4) $\left.\frac{1}{2} \nabla \cdot \mathbf{w}\right|_{K}+\left.r\right|_{K} \geq c_{\mathbf{w}, r, K}$ and $|\nabla \cdot \mathbf{w}|_{K}+\left.r\right|_{K} \mid \leq C_{\mathbf{w}, r, K}, c_{\mathbf{w}, r, K} \geq 0, C_{\mathbf{w}, r, K} \geq 0$, for all $K \in \widetilde{\mathcal{T}}_{h}$;

(B5) $f$ is a polynomial of degree at most $k$ on each $K \in \widetilde{\mathcal{T}}_{h}$;

(B6) $g \in \mathrm{H}^{1 / 2}\left(\Gamma_{\mathrm{D}}\right)$;

(B7) $u \in \mathrm{L}^{2}\left(\Gamma_{\mathrm{N}}\right)$;

(B8) if $c_{\mathbf{w}, r, K}=0$, then $C_{\mathbf{w}, r, K}=0$.

The assumptions on $\mathbf{S}, r, f$, and $\mathbf{w}$ (cf. Section 3.2 below for the definition of the space $\mathbf{R T}\left(\widetilde{\mathcal{T}}_{h}\right)$ ) are made for the sake of simplicity and are usually satisfied in practice. If the functions at hand do not fulfill the given requirements, interpolation can be used. Also, note that Assumption (B8) allows $c_{\mathbf{w}, r, K}=0$ but $\left.\mathbf{w}\right|_{K} \neq 0$. 


\subsection{Continuous problem}

Let $\mathcal{T}_{h}$ be, as throughout the whole paper, a refinement of $\widetilde{\mathcal{T}}_{h}$. We define a bilinear form $\mathcal{B}$ by

$$
\mathcal{B}(p, \varphi):=\sum_{K \in \mathcal{T}_{h}}\left\{(\mathbf{S} \nabla p, \nabla \varphi)_{K}+(\nabla \cdot(p \mathbf{w}), \varphi)_{K}+(r p, \varphi)_{K}\right\} \quad p, \varphi \in \mathrm{H}^{1}\left(\mathcal{T}_{h}\right),
$$

where $\mathrm{H}^{1}\left(\mathcal{T}_{h}\right)$ is the "broken Sobolev space", $\mathrm{H}^{1}\left(\mathcal{T}_{h}\right):=\left\{\varphi \in \mathrm{L}^{2}(\Omega) ;\left.\varphi\right|_{K} \in \mathrm{H}^{1}(K) \forall K \in \mathcal{T}_{h}\right\}$, and the corresponding energy (semi-)norm by

$$
\|\varphi\|_{\Omega}^{2}:=\sum_{K \in \mathcal{T}_{h}}\|\varphi\|_{K}^{2}, \quad\|\varphi\|_{K}^{2}:=\left\|\mathbf{S}^{\frac{1}{2}} \nabla \varphi\right\|_{K}^{2}+\left\|\left(\frac{1}{2} \nabla \cdot \mathbf{w}+r\right)^{\frac{1}{2}} \varphi\right\|_{K}^{2} \quad \varphi \in \mathrm{H}^{1}\left(\mathcal{T}_{h}\right) .
$$

In this way $\mathcal{B}(\cdot, \cdot)$ and $\|\mid \cdot\|_{\Omega}$ are well-defined for $p, \varphi \in \mathrm{H}^{1}(\Omega)$ as well as for $p, \varphi$ that are only piecewise regular. We remark that $\left|\|\cdot \mid\|_{\Omega}\right.$ is always a norm on $\mathrm{H}_{\mathrm{D}}^{1}(\Omega)$, the subspace of $\mathrm{H}^{1}(\Omega)$ of functions with traces vanishing on $\Gamma_{\mathrm{D}}$, whereas it is a norm on $\mathrm{H}^{1}\left(\mathcal{T}_{h}\right)$ only when $c_{\mathrm{w}, r, K}>0$ for all $K \in \mathcal{T}_{h}$. The weak formulation of the problem (1.1a)-(1.1c) is then to find $p \in \mathrm{H}^{1}(\Omega)$ with $\left.p\right|_{\Gamma_{\mathrm{D}}}=g$ in the sense of traces such that

$$
\mathcal{B}(p, \varphi)=(f, \varphi)_{\Omega}-\langle u, \varphi\rangle_{\Gamma_{\mathrm{N}}} \quad \forall \varphi \in \mathrm{H}_{\mathrm{D}}^{1}(\Omega)
$$

Problem (2.6) under Assumption (B) in particular admits a unique solution.

\section{Finite volume And miXed Finite Element SCHemes And Postprocessing}

We first introduce here the finite volume and mixed finite element methods for the problem (1.1a)-(1.1c). Since the original approximations in these methods are only piecewise constant and thus lack regularity so as to be directly used in an a posteriori error estimate, we next construct a locally postprocessed approximation $\tilde{p}_{h}$, also based on the known fluxes. Finally, since $\tilde{p}_{h}$ will be nonconforming, we will need its Oswald interpolate later.

\subsection{The finite volume method}

A general cell-centered finite volume scheme for the problem (1.1a)-(1.1c) can be written in the following form: find $p_{K}, K \in \mathcal{T}_{h}$, the approximations to $p$ such that

$$
\sum_{\sigma \in \mathcal{E}_{K}} S_{K, \sigma}+\sum_{\sigma \in \mathcal{E}_{K}} W_{K, \sigma}+r_{K} p_{K}|K|=f_{K}|K| \quad \forall K \in \mathcal{T}_{h}
$$

where $f_{K}:=(f, 1) /|K|, r_{K}:=(r, 1) /|K|$, and where $S_{K, \sigma}$ and $W_{K, \sigma}$ are, respectively, the diffusive and convective fluxes through the sides $\sigma$ of an element $K$, functions of $p_{K}, K \in \mathcal{T}_{h}$, of the mesh, and of the data. For the a posteriori error estimates presented in this paper, we do not need the specific form of the diffusive and convective fluxes; our analysis however relies on the finite volume concept of their continuity, imposing that $S_{K, \sigma_{K, L}}=-S_{L, \sigma_{K, L}}$ and $W_{K, \sigma_{K, L}}=-W_{L, \sigma_{K, L}}$ for all $\sigma_{K, L} \in \mathcal{E}_{h}^{\text {int }}$. To fix ideas, we give an example.

When $\mathcal{T}_{h}$ is admissible in the sense of [10, Definition 9.1] (when there exist points $\mathbf{x}_{K}$ in the interior of each $K \in \mathcal{T}_{h}$ such that the straight lines connecting $\mathbf{x}_{K}$ and $\mathbf{x}_{L}$ for two neighboring elements $K$ and $L$ are orthogonal to $\sigma_{K, L}$ and when an analogous orthogonality condition holds on the Dirichlet part of the boundary), and under 
the additional assumption that $\mathbf{S}_{K}=s_{K} I d$ for all $K \in \mathcal{T}_{h}$, the following choices for $S_{K, \sigma}$ and $W_{K, \sigma}$ are possible:

$$
\begin{aligned}
S_{K, \sigma} & =-s_{K, L} \frac{\left|\sigma_{K, L}\right|}{d_{K, L}}\left(p_{L}-p_{K}\right) \quad \sigma=\sigma_{K, L} \in \mathcal{E}_{h}^{\mathrm{int}}, \\
S_{K, \sigma} & =-s_{K} \frac{|\sigma|}{d_{K, \sigma}}\left(g_{\sigma}-p_{K}\right) \quad \sigma \in \mathcal{E}_{K} \cap \mathcal{E}_{h}^{\mathrm{D}}, \\
S_{K, \sigma} & =u_{\sigma}|\sigma| \quad \sigma \in \mathcal{E}_{K} \cap \mathcal{E}_{h}^{\mathrm{N}}, \\
W_{K, \sigma} & =p_{\sigma} w_{K, \sigma} \quad \sigma \in \mathcal{E}_{K} .
\end{aligned}
$$

Here $d_{K, L}=\left|\mathbf{x}_{K}-\mathbf{x}_{L}\right|$, the Euclidean distance of $\mathbf{x}_{K}$ and $\mathbf{x}_{L}, d_{K, \sigma}$ is the Euclidean distance of $\mathbf{x}_{K}$ and $\sigma \in \mathcal{E}_{K} \cap \mathcal{E}_{h}^{\mathrm{D}}$, and

$$
g_{\sigma}:=\frac{\langle g, 1\rangle_{\sigma}}{|\sigma|}, \quad u_{\sigma}:=\frac{\langle u, 1\rangle_{\sigma}}{|\sigma|}, \quad w_{K, \sigma}:=\langle\mathbf{w} \cdot \mathbf{n}, 1\rangle_{\sigma} .
$$

For the value $s_{K, L}$, we can choose either an arithmetic or a harmonic averaging in the diffusion-dispersion tensor $-s_{K, L}:=\left(s_{K}+s_{L}\right) / 2$ or $s_{K, L}:=2 s_{K} s_{L} /\left(s_{K}+s_{L}\right)$. Finally, $p_{\sigma}$ can be e.g. a weighted upwind value, comprised between $p_{K}$ and $p_{L}$ for $\sigma_{K, L} \in \mathcal{E}_{h}^{\text {int }}$, see [24,23] for details. For other schemes, including those enabling to take into account a general $\mathbf{S}$, cf. $[1,7,12]$.

\subsection{The lowest-order Raviart-Thomas mixed finite element method}

Let us denote by $\mathbf{R T}\left(\mathcal{T}_{h}\right)$ the space of elementwise linear vector functions $\mathbf{u}_{h}$ such that, on each $K \in \mathcal{T}_{h}$, $\left.\mathbf{u}_{h}\right|_{K}=\left(a_{K}+d_{K} x, b_{K}+d_{K} y\right)$ if $d=2$ and $\left.\mathbf{u}_{h}\right|_{K}=\left(a_{K}+d_{K} x, b_{K}+d_{K} y, c_{K}+d_{K} z\right)$ if $d=3$, with the normal component continuous over the interior sides. Let $\mathbf{R} \mathbf{T}_{\mathrm{N}}\left(\mathcal{T}_{h}\right)$ be its subspace of functions satisfying $\left.\left(\mathbf{u}_{h} \cdot \mathbf{n}\right)\right|_{\sigma}=u_{\sigma}$ for all $\sigma \in \mathcal{E}_{h}^{\mathrm{N}}$. Here $u_{\sigma}$ is given by (3.6) (recall that the normal components of $\mathbf{u}_{h} \in \mathbf{R T}\left(\mathcal{T}_{h}\right)$ are constant on each $\sigma \in \mathcal{E}_{h}$ and that they represent the degrees of freedom of $\mathbf{R T}\left(\mathcal{T}_{h}\right)$ ). Let finally $\mathbf{R T}_{0, \mathrm{~N}}\left(\mathcal{T}_{h}\right)$ be such that $\left.\left(\mathbf{u}_{h} \cdot \mathbf{n}\right)\right|_{\sigma}=0$ for all $\sigma \in \mathcal{E}_{h}^{\mathrm{N}}$.

Under the above definitions, the centered mixed finite element scheme (cf. $[9,18]$ ) reads: find $\mathbf{u}_{h} \in \mathbf{R} \mathbf{T}_{\mathrm{N}}\left(\mathcal{T}_{h}\right)$ and $p_{h}$ given by $p_{K}$ on each $K \in \mathcal{T}_{h}$ such that

$$
\begin{gathered}
\left(\mathbf{S}^{-1} \mathbf{u}_{h}, \mathbf{v}_{h}\right)_{\Omega}-\left(p_{h}, \nabla \cdot \mathbf{v}_{h}\right)_{\Omega}=-\left\langle\mathbf{v}_{h} \cdot \mathbf{n}, g\right\rangle_{\Gamma_{\mathrm{D}}} \quad \forall \mathbf{v}_{h} \in \mathbf{R T}_{0, \mathrm{~N}}\left(\mathcal{T}_{h}\right), \\
\left(\nabla \cdot \mathbf{u}_{h}, 1\right)_{K}-\left(\mathbf{S}^{-1} \mathbf{u}_{h} \cdot \mathbf{w}, 1\right)_{K}+\left((r+\nabla \cdot \mathbf{w}) p_{K}, 1\right)_{K}=(f, 1)_{K} \quad \forall K \in \mathcal{T}_{h},
\end{gathered}
$$

whereas the upwind-weighted mixed finite element scheme (cf. [8]) reads: find $\mathbf{u}_{h} \in \mathbf{R} \mathbf{T}_{\mathrm{N}}\left(\mathcal{T}_{h}\right)$ and $p_{K}$ given by $p_{K}$ on each $K \in \mathcal{T}_{h}$ such that (with $W_{K, \sigma}$ given by (3.5)-(3.6))

$$
\begin{gathered}
\left(\mathbf{S}^{-1} \mathbf{u}_{h}, \mathbf{v}_{h}\right)_{\Omega}-\left(p_{h}, \nabla \cdot \mathbf{v}_{h}\right)_{\Omega}=-\left\langle\mathbf{v}_{h} \cdot \mathbf{n}, g\right\rangle_{\Gamma_{\mathrm{D}}} \quad \forall \mathbf{v}_{h} \in \mathbf{R} \mathbf{T}_{0, \mathrm{~N}}\left(\mathcal{T}_{h}\right), \\
\left(\nabla \cdot \mathbf{u}_{h}, 1\right)_{K}+\sum_{\sigma \in \mathcal{E}_{K}} W_{K, \sigma}+\left(r p_{K}, 1\right)_{K}=(f, 1)_{K} \quad \forall K \in \mathcal{T}_{h} .
\end{gathered}
$$

\subsection{Postprocessing}

Let $\mathbf{u}_{h} \in \mathbf{R T}_{\mathrm{N}}\left(\mathcal{T}_{h}\right)$ be given by (3.7a)-(3.7b) or (3.8a)-(3.8b) in the mixed finite element method. Similarly, let $\mathbf{u}_{h} \in \mathbf{R} \mathbf{T}_{\mathrm{N}}\left(\mathcal{T}_{h}\right)$ be prescribed by the finite volume fluxes $S_{K, \sigma}(3.2)-(3.4)$, i.e. on each $K \in \mathcal{T}_{h}$, let $\left.\mathbf{u}_{h}\right|_{K}$ be such that $\left.\left(\mathbf{u}_{h} \cdot \mathbf{n}\right)\right|_{\sigma}=S_{K, \sigma} /|\sigma|$, cf. [11]. We then define a postprocessed approximation $\tilde{p}_{h}$ on each simplex in the following way:

$$
\begin{aligned}
-\left.\mathbf{S}_{K} \nabla \tilde{p}_{h}\right|_{K} & =\left.\mathbf{u}_{h}\right|_{K} \quad \forall K \in \mathcal{T}_{h}, \\
\left(1-\mu_{K}\right) \frac{\left(\tilde{p}_{h}, 1\right)_{K}}{|K|}+\mu_{K} \tilde{p}_{h}\left(\mathbf{x}_{K}\right) & =p_{K} \quad \forall K \in \mathcal{T}_{h} .
\end{aligned}
$$


Here $\mu_{K}=0$ for mixed finite elements and $\mu_{K}=0$ or 1 for finite volumes, in the dependence on whether the particular scheme (3.1) represents by $p_{K}$ the approximate mean or point value (we for simplicity assume that $\left.\mathbf{x}_{K} \in K\right)$. It is immediate to show that such $\tilde{p}_{h}$ exists, is unique, writes as a second-order polynomial on each $K \in \mathcal{T}_{h}$, but is nonconforming (not included in $\mathrm{H}^{1}(\Omega)$ ). However, for mixed finite elements, it is shown in [24] that at least the means of traces over interior sides are continuous. Also, convergence and a priori error estimates for $\tilde{p}_{h}$ in the finite volume case, under the condition that the original finite volume scheme satisfies some necessary properties, are proved in [23]. Note finally that the proposed postprocessing is local on each element and that one only has to build a second-order polynomial on each $K \in \mathcal{T}_{h}$ from the prescribed degrees of freedom, so that its cost is negligible.

\subsection{Oswald interpolation operator}

Let $\mathbb{P}_{l}\left(\mathcal{T}_{h}\right)$ denote the space of polynomials of total degree at most $l$ on each simplex. The Oswald interpolation operator $\mathcal{I}_{\mathrm{Os}}: \mathbb{P}_{l}\left(\mathcal{T}_{h}\right) \rightarrow \mathbb{P}_{l}\left(\mathcal{T}_{h}\right) \cap \mathrm{H}^{1}(\Omega)$ is defined as follows: given a function $\varphi_{h} \in \mathbb{P}_{l}\left(\mathcal{T}_{h}\right)$, the value of $\mathcal{I}_{\mathrm{Os}}\left(\varphi_{h}\right)$ is prescribed at the Lagrangian nodes of $\mathbb{P}_{l}\left(\mathcal{T}_{h}\right) \cap \mathrm{H}^{1}(\Omega)$ by the average of the values of $\varphi_{h}$ at this node. We finally define $\mathcal{I}_{\mathrm{Os}}^{\Gamma}\left(\tilde{p}_{h}\right) \in \mathrm{H}^{1}(\Omega)$, differing from $\mathcal{I}_{\mathrm{Os}}\left(\tilde{p}_{h}\right)$ only on such $K \in \mathcal{T}_{h}$ that contain a boundary side, by

$$
\begin{aligned}
& \left.\mathcal{I}_{\mathrm{Os}}^{\Gamma}\left(\tilde{p}_{h}\right)\right|_{\Gamma_{\mathrm{D}}}=g \quad \text { in the sense of traces, } \\
& \left\langle\mathcal{I}_{\mathrm{Os}}^{\Gamma}\left(\tilde{p}_{h}\right) \mathbf{w} \cdot \mathbf{n}, 1\right\rangle_{\sigma}=W_{K, \sigma} \quad \forall \sigma \in \mathcal{E}_{h}^{\mathrm{N}},
\end{aligned}
$$

where we put $W_{K, \sigma}=\left\langle\tilde{p}_{h} \mathbf{w} \cdot \mathbf{n}, 1\right\rangle_{\sigma}$ for the centered mixed finite element scheme (3.7a)-(3.7b). Note that if $\mathbf{w} \cdot \mathbf{n}$ is constant but nonzero on a given $\sigma \in \mathcal{E}_{h}^{\mathrm{N}}$, then (3.10b) prescribes the mean value of $\mathcal{I}_{\text {Os }}^{\Gamma}\left(\tilde{p}_{h}\right)$ on this side by $W_{K, \sigma} /\langle\mathbf{w} \cdot \mathbf{n}, 1\rangle_{\sigma}$, i.e. by $p_{\sigma}$ for the scheme (3.5)-(3.6).

\section{A posteriori ERror estimate}

We give in this section our a posteriori estimate as well as a sketch of its proof.

\subsection{A posteriori error estimate}

Let us first put

$$
m_{K}^{2}:=\min \left\{C_{\mathrm{P}} \frac{h_{K}^{2}}{c_{\mathbf{S}, K}}, \frac{1}{c_{\mathbf{w}, r, K}}\right\}
$$

for all $K \in \mathcal{T}_{h}$, where $C_{\mathrm{P}}$ is the constant from the Poincaré inequality (2.1). We define the residual estimator $\eta_{\mathrm{R}, K}$ by

$$
\eta_{\mathrm{R}, K}:=m_{K}\left\|f+\nabla \cdot\left(\mathbf{S} \nabla \tilde{p}_{h}\right)-\nabla \cdot\left(\tilde{p}_{h} \mathbf{w}\right)-r \tilde{p}_{h}\right\|_{K} .
$$

Let $\mathcal{I}_{\mathrm{Os}}^{\Gamma}\left(\tilde{p}_{h}\right)$ be the modification of the Oswald interpolate $\mathcal{I}_{\mathrm{Os}}\left(\tilde{p}_{h}\right)$ at the boundary given by (3.10a)-(3.10b). Denote $v:=\tilde{p}_{h}-\mathcal{I}_{\mathrm{Os}}^{\Gamma}\left(\tilde{p}_{h}\right)$. Then the nonconformity estimator $\eta_{\mathrm{NC}, K}$ is given by

$$
\eta_{\mathrm{NC}, K}:=\mid\|v\|_{K}
$$

and the convection estimator $\eta_{\mathrm{C}, K}$ by

$$
\begin{aligned}
\eta_{\mathrm{C}, K}:= & \min \left\{\frac{\left\|\nabla \cdot(v \mathbf{w})-\frac{1}{2} v \nabla \cdot \mathbf{w}\right\|_{K}+\|\nabla \cdot(v \mathbf{w})\|_{K}}{\sqrt{c_{\mathbf{w}, r, K}}},\right. \\
& \left.\left(\frac{C_{\mathrm{P}} h_{K}^{2}\|\nabla v \cdot \mathbf{w}\|_{K}^{2}}{c_{\mathbf{S}, K}}+\frac{9\|v \nabla \cdot \mathbf{w}\|_{K}^{2}}{4 c_{\mathbf{w}, r, K}}\right)^{\frac{1}{2}}\right\} .
\end{aligned}
$$

Next, let

$$
m_{\sigma}^{2}:=\min \left\{\max _{K ; \sigma \in \mathcal{E}_{K}}\left\{C_{\mathrm{F}, d} \frac{|\sigma| h_{K}^{2}}{|K| c_{\mathbf{S}, K}}\right\}, \max _{K ; \sigma \in \mathcal{E}_{K}}\left\{\frac{|\sigma|}{|K| c_{\mathbf{w}, r, K}}\right\}\right\}
$$


for interior or Dirichlet boundary sides. Here $C_{\mathrm{F}, d}$ is the constant from the generalized Friedrichs inequality $(2.2)$. The upwinding estimator $\eta_{\mathrm{U}, K}$ is given by

$$
\eta_{\mathrm{U}, K}:=\sum_{\sigma \in \mathcal{E}_{K} \backslash \mathcal{E}_{h}^{\mathrm{N}}} m_{\sigma}\left\|\left(W_{K, \sigma}-\left\langle\mathcal{I}_{\mathrm{O}}^{\Gamma}\left(\tilde{p}_{h}\right) \mathbf{w} \cdot \mathbf{n}, 1\right\rangle_{\sigma}\right)|\sigma|^{-1}\right\|_{\sigma}
$$

Finally, the reaction quadrature estimator $\eta_{\mathrm{RQ}, K}$ is given by

$$
\eta_{\mathrm{RQ}, K}:=\frac{1}{\sqrt{c_{\mathrm{w}, r, K}}}\left\|r_{K} p_{K}-\left(r \tilde{p}_{h}, 1\right)_{K}|K|^{-1}\right\|_{K}
$$

and the Neumann boundary estimator $\eta_{\Gamma_{\mathrm{N}}, K}$ by

$$
\eta_{\Gamma_{\mathrm{N}}, K}:=0+\frac{1}{\sqrt{{ }^{c_{\mathbf{S}, K}}}} \sum_{\sigma \in \mathcal{E}_{K} \cap \mathcal{E}_{h}^{\mathrm{N}}} \sqrt{C_{\mathrm{t}, K, \sigma}} \sqrt{h_{K}}\left\|u_{\sigma}-u\right\|_{\sigma},
$$

where $C_{\mathrm{t}, K, \sigma}$ is the constant from the trace inequality (2.3). We then have the following estimate:

Theorem 4.1 (A posteriori error estimate). Let $p$ be the weak solution of the problem (1.1a)-(1.1c) given by (2.6). Let a finite volume scheme of the form (3.1), with the diffusive fluxes through Neumann boundary sides prescribed by (3.4), (3.6), or a mixed finite element scheme (3.7a)-(3.7b) or (3.8a)-(3.8b), be given. Let $\tilde{p}_{h}$ be the postprocessed solution given by (3.9a)-(3.9b). Then

$$
\left\|p-\tilde{p}_{h}\right\|_{\Omega} \leq\left\{\sum_{K \in \mathcal{T}_{h}} \eta_{\mathrm{NC}, K}^{2}\right\}^{1 / 2}+\left\{\sum_{K \in \mathcal{T}_{h}}\left(\eta_{\mathrm{R}, K}+\eta_{\mathrm{C}, K}+\eta_{\mathrm{U}, K}+\eta_{\mathrm{RQ}, K}+\eta_{\Gamma_{\mathrm{N}}, K}\right)^{2}\right\}^{1 / 2} .
$$

\subsection{Sketch of the proof of the estimate}

We first remark that using the triangle inequality and the coercivity of the form $\mathcal{B}(\cdot, \cdot)$ given by $\|\varphi \mid\|_{\Omega}^{2} \leq$ $\mathcal{B}(\varphi, \varphi)$ for all $\varphi \in \mathrm{H}_{\mathrm{D}}^{1}(\Omega)$, we have, for $p, s \in \mathrm{H}^{1}(\Omega)$ such that $p-s \in \mathrm{H}_{\mathrm{D}}^{1}(\Omega)$ and an arbitrary $\tilde{p} \in \mathrm{H}^{1}\left(\mathcal{T}_{h}\right)$ (to simplify notation, we use the convention $0 / 0=0)$,

$$
\begin{aligned}
\||| p-\tilde{p}\|_{\Omega} & \leq\|\tilde{p}-s\|_{\Omega}+\|p-s\|_{\Omega} \leq\|\tilde{p}-s\|_{\Omega}+\frac{\mathcal{B}(p-s, p-s)}{\left.\|p-s\|\right|_{\Omega}} \\
& =\|\tilde{p}-s\|_{\Omega}+\mathcal{B}\left(p-\tilde{p}, \frac{p-s}{\|p-s\|_{\Omega}}\right)+\mathcal{B}\left(\tilde{p}-s, \frac{p-s}{\|p-s\|_{\Omega}}\right) .
\end{aligned}
$$

The following lemma, whose proof can be found in [24], gives an important improvement of the above bound, allowing in particular for the asymptotic exactness of Section 5.2 below:

Lemma 4.2 (Abstract framework). Let $p, s \in \mathrm{H}^{1}(\Omega), p-s \in \mathrm{H}_{\mathrm{D}}^{1}(\Omega)$, and let $\tilde{p} \in \mathrm{H}^{1}\left(\mathcal{T}_{h}\right)$. Then

$$
\begin{aligned}
\|p-\tilde{p}\|_{\Omega} \leq & \|\tilde{p}-s\|_{\Omega}+\mid \mathcal{B}\left(p-\tilde{p}, \frac{p-s}{\|\mid p-s\|_{\Omega}}\right)+ \\
& \sum_{K \in \mathcal{T}_{h}}\left(\nabla \cdot((\tilde{p}-s) \mathbf{w})-\frac{1}{2}(\tilde{p}-s) \nabla \cdot \mathbf{w}, \frac{p-s}{\|p-s\|_{\Omega}}\right)_{K} \mid .
\end{aligned}
$$

Consequently, the following bound for the error $\left\|\mid p-\tilde{p}_{h}\right\|_{\Omega}$ holds: 
Lemma 4.3 (Abstract error estimate). Let the assumptions of Theorem 4.1 be verified and let $s \in \mathrm{H}^{1}(\Omega)$ such that $\left.s\right|_{\Gamma_{\mathrm{D}}}=g$ in the sense of traces be arbitrary. Then

$$
\left\|p-\tilde{p}_{h}\right\|_{\Omega} \leq\left\|\tilde{p}_{h}-s\right\|_{\Omega}+\sup _{\varphi \in \mathrm{H}_{\mathrm{D}}^{1}(\Omega),\|\varphi\|_{\Omega}=1}\left\{T_{\mathrm{R}}(\varphi)+T_{\mathrm{C}}(\varphi)+T_{\mathrm{U}}(\varphi)+T_{\mathrm{RQ}}(\varphi)+T_{\Gamma_{\mathrm{N}}}(\varphi)\right\},
$$

where, putting $\varphi_{K}:=(\varphi, 1)_{K} /|K|$ and $\varphi_{\sigma}:=\langle\varphi, 1\rangle_{\sigma} /|\sigma|$,

$$
\begin{aligned}
T_{\mathrm{R}}(\varphi) & :=\sum_{K \in \mathcal{T}_{h}}\left(f+\nabla \cdot\left(\mathbf{S} \nabla \tilde{p}_{h}\right)-\nabla \cdot\left(\tilde{p}_{h} \mathbf{w}\right)-r \tilde{p}_{h}, \varphi-\varphi_{K}\right)_{K}, \\
T_{\mathrm{C}}(\varphi) & :=\sum_{K \in \mathcal{T}_{h}}\left\{\left(\nabla \cdot\left(\left(\tilde{p}_{h}-s\right) \mathbf{w}\right)-\frac{1}{2}\left(\tilde{p}_{h}-s\right) \nabla \cdot \mathbf{w}, \varphi\right)_{K}+\left\langle\left(s-\tilde{p}_{h}\right) \mathbf{w} \cdot \mathbf{n}, \varphi_{K}\right\rangle_{\partial K}\right\}, \\
T_{\mathrm{U}}(\varphi) & :=\sum_{K \in \mathcal{T}_{h}} \sum_{\sigma \in \mathcal{E}_{K}}\left\langle\left(W_{K, \sigma}-\langle s \mathbf{w} \cdot \mathbf{n}, 1\rangle_{\sigma}\right)|\sigma|^{-1}, \varphi_{K}\right\rangle_{\sigma}, \\
T_{\mathrm{RQ}}(\varphi) & :=\sum_{K \in \mathcal{T}_{h}}\left(r_{K} p_{K}-\left(r \tilde{p}_{h}, 1\right)_{K}|K|^{-1}, \varphi_{K}\right)_{K}, \\
T_{\Gamma_{\mathrm{N}}}(\varphi) & :=\sum_{\sigma \in \mathcal{E}_{h}^{\mathrm{N}}}\left\langle u_{\sigma}-u, \varphi-\varphi_{\sigma}\right\rangle_{\sigma} .
\end{aligned}
$$

Proof. Let us consider an arbitrary $\varphi \in \mathrm{H}_{\mathrm{D}}^{1}(\Omega)$. We have, using the bilinearity of $\mathcal{B}(\cdot, \cdot)$, the definition $(2.6)$ of the weak solution $p$, and the Green theorem in each $K \in \mathcal{T}_{h}$,

$$
\begin{aligned}
\mathcal{B}\left(p-\tilde{p}_{h}, \varphi\right) & =(f, \varphi)_{\Omega}-\langle u, \varphi\rangle_{\Gamma_{\mathrm{N}}}-\sum_{K \in \mathcal{T}_{h}}\left\{\left(\mathbf{S} \nabla \tilde{p}_{h}, \nabla \varphi\right)_{K}+\left(\nabla \cdot\left(\tilde{p}_{h} \mathbf{w}\right), \varphi\right)_{K}+\left(r \tilde{p}_{h}, \varphi\right)_{K}\right\} \\
& =\sum_{K \in \mathcal{T}_{h}}\left(f+\nabla \cdot\left(\mathbf{S} \nabla \tilde{p}_{h}\right)-\nabla \cdot\left(\tilde{p}_{h} \mathbf{w}\right)-r \tilde{p}_{h}, \varphi\right)_{K}-\sum_{K \in \mathcal{T}_{h}}\left\langle\mathbf{S} \nabla \tilde{p}_{h} \cdot \mathbf{n}, \varphi\right\rangle_{\partial K}-\langle u, \varphi\rangle_{\Gamma_{\mathrm{N}}} \\
& =\sum_{K \in \mathcal{T}_{h}}\left\{\left(f+\nabla \cdot\left(\mathbf{S} \nabla \tilde{p}_{h}\right)-\nabla \cdot\left(\tilde{p}_{h} \mathbf{w}\right)-r \tilde{p}_{h}, \varphi\right)_{K}+\sum_{\sigma \in \mathcal{E}_{K} \cap \Gamma_{\mathrm{N}}}\left\langle u_{\sigma}-u, \varphi\right\rangle_{\sigma}\right\} .
\end{aligned}
$$

Note that we have in particular used the continuity of the normal trace of $\mathbf{S} \nabla \tilde{p}_{h}$ across interior sides following from finite volume or mixed finite element fluxes continuity, i.e.

$$
\left\langle\left.\left(\mathbf{S} \nabla \tilde{p}_{h} \cdot \mathbf{n}\right)\right|_{K}+\left.\left(\mathbf{S} \nabla \tilde{p}_{h} \cdot \mathbf{n}\right)\right|_{L}, \varphi\right\rangle_{\sigma_{K, L}}=\langle 0, \varphi\rangle_{\sigma_{K, L}}=0 \quad \forall \sigma_{K, L} \in \mathcal{E}_{h}^{\text {int }},
$$

the fact that $\left\langle\mathbf{S} \nabla \tilde{p}_{h} \cdot \mathbf{n}, \varphi\right\rangle_{\sigma}=0$ for $\sigma \in \mathcal{E}_{h}^{\mathrm{D}}$ following by $\varphi \in \mathrm{H}_{\mathrm{D}}^{1}(\Omega)$, and finally (3.4) for Neumann boundary sides. If $\sigma \in \mathcal{E}_{h}^{\mathrm{N}}$, notice moreover that $\left\langle u_{\sigma}-u, \varphi\right\rangle_{\sigma}=\left\langle u_{\sigma}-u, \varphi-\varphi_{\sigma}\right\rangle_{\sigma}$, since $\left\langle u_{\sigma}-u, \varphi_{\sigma}\right\rangle_{\sigma}=0$ by (3.6) (recall that $\varphi_{\sigma}$ is a constant).

Now by the definition (3.1) of the finite volume scheme, or by (3.7b) or (3.8b) in the mixed finite element case, it follows that

$$
\begin{aligned}
& \left(f+\nabla \cdot\left(\mathbf{S} \nabla \tilde{p}_{h}\right)-\nabla \cdot\left(\tilde{p}_{h} \mathbf{w}\right)-r \tilde{p}_{h}, \varphi_{K}\right)_{K}+\left\langle\tilde{p}_{h} \mathbf{w} \cdot \mathbf{n}, \varphi_{K}\right\rangle_{\partial K}-\varphi_{K} \sum_{\sigma \in \mathcal{E}_{K}} W_{K, \sigma} \\
& +\left(r \tilde{p}_{h}, \varphi_{K}\right)_{K}-\varphi_{K} r_{K} p_{K}|K| \pm\left\langle s \mathbf{w} \cdot \mathbf{n}, \varphi_{K}\right\rangle_{\partial K}=0 \quad \forall K \in \mathcal{T}_{h} .
\end{aligned}
$$

To see this, recall that $\varphi_{K}$ is the constant mean of $\varphi$ over $K$, that $\left(\nabla \cdot\left(\mathbf{S} \nabla \tilde{p}_{h}\right), 1\right)_{K}=\left\langle\mathbf{S} \nabla \tilde{p}_{h} \cdot \mathbf{n}, 1\right\rangle_{\partial K}=$ $-\sum_{\sigma \in \mathcal{E}_{K}} S_{K, \sigma}$ by the Green theorem in the finite volume case, and that

$$
-\left(\mathbf{S}_{K}^{-1} \mathbf{u}_{h} \cdot \mathbf{w}, 1\right)_{K}+\left(p_{K} \nabla \cdot \mathbf{w}, 1\right)_{K}=\left(\nabla \tilde{p}_{h} \cdot \mathbf{w}, 1\right)_{K}+\left(\tilde{p}_{h} \nabla \cdot \mathbf{w}, 1\right)_{K}=\left\langle\tilde{p}_{h} \mathbf{w} \cdot \mathbf{n}, 1\right\rangle_{\partial K},
$$


using that $\mathbf{w} \in \mathbf{R T}\left(\mathcal{T}_{h}\right)$ by Assumption (B2) in the centered mixed finite element one. Hence we can subtract this term from each summand in (4.4). To conclude the proof, if now suffices to use Lemma 4.2.

Theorem 4.1 can now be proved by estimating the terms $T_{\mathrm{R}}, T_{\mathrm{C}}, T_{\mathrm{U}}, T_{\mathrm{RQ}}$, and $T_{\Gamma_{\mathrm{N}}}$ separately, putting $s=\mathcal{I}_{\mathrm{Os}}^{\Gamma}\left(\tilde{p}_{h}\right)$ in Lemma 4.3 and using the Schwarz and Cauchy-Schwarz inequalities, the Poincaré inequality (2.1), the generalized Friedrichs inequality (2.2), the trace inequality (2.3), (3.10b), the Green theorem, and the definition of \|\|$\cdot\|\|_{K}$ by $(2.5)$. It is detailed in [24,23].

\section{VARIOUS REMARKS}

We give several remarks in this section.

\subsection{Nature of the estimates and some comments on the proof}

The basis for our a posteriori error estimates is the construction of the postprocessed approximation $\tilde{p}_{h}$, which is then compared to the primal weak solution (2.6) in (4.4), as in the finite element method, cf. [20]. The finite element Galerkin orthogonality is replaced by the element conservativity equations (3.1) and (3.7b) or (3.8b), respectively, which yields (4.5). Consequently, residual estimators similar to those in the finite element case are obtained. However, whereas in the lowest-order Galerkin finite element method, $\left.\nabla \cdot\left(\mathbf{S}_{K} \nabla p_{h}\right)\right|_{K}$ is always equal to zero on all $K \in \mathcal{T}_{h}$, our element residuals $\eta_{\mathrm{R}, K}$ (4.1) are relevant even if the original finite volume or mixed finite element solution is elementwise constant. The crucial point is however that in contrast to Galerkin finite element approximations, the normal traces of $-\mathbf{S} \nabla \tilde{p}_{h}=\mathbf{u}_{h}$ are continuous across interior sides in finite volume or mixed finite element schemes. Hence the side error estimators penalizing the mass balance common in Galerkin finite element methods (cf. [20]) do not appear here at all. However, $\tilde{p}_{h}$ is nonconforming in the sense that it is not included in $\mathrm{H}^{1}(\Omega)$, so that the estimator $\eta_{\mathrm{NC}, K}(4.2)$ comes in place.

\subsection{Asymptotic exactness and asymptotic robustness with respect to inhomogeneities and anisotropies}

For pure diffusion problems (i.e. $\mathbf{w}=r=0$ in (1.1a)-(1.1c)), using the fact that $s \in \mathrm{H}^{1}(\Omega)$ such that $\left.s\right|_{\Gamma_{\mathrm{D}}}=g$ in Lemma 4.3 was chosen arbitrarily, it shows that we in fact have

$$
||\left|p-\tilde{p}_{h}\right|\left\|_{\Omega} \leq \inf _{s \in \mathrm{H}^{1}(\Omega),\left.s\right|_{\Gamma_{\mathrm{D}}}=g}\right\|\left|\tilde{p}_{h}-s\right| \|_{\Omega}+\left\{\sum_{K \in \mathcal{T}_{h}}\left(\eta_{\mathrm{R}, K}+\eta_{\Gamma_{\mathrm{N}}, K}\right)^{2}\right\}^{1 / 2} .
$$

Moreover, in this case, $-\nabla \cdot\left(\mathbf{S}_{K} \nabla \tilde{p}_{h}\right)=f_{K}$ for all $K \in \mathcal{T}_{h}$ using (3.9a) and (3.1) or (3.7b) or (3.8b). This by virtue of

$$
\inf _{s \in \mathrm{H}^{1}(\Omega),\left.s\right|_{\Gamma_{\mathrm{D}}}=g}\left\|\left|\tilde{p}_{h}-s\right|\right\|_{\Omega} \leq\left\|\mid \tilde{p}_{h}-p\right\| \|_{\Omega}
$$

shows that such an estimate is asymptotically exact (asymptotically equal to the error) and asymptotically fully robust with respect to inhomogeneities and anisotropies (asymptotically with respect to the approximation of $\tilde{p}_{h}$ by some $s \in \mathrm{H}^{1}(\Omega)$ such that $\left.s\right|_{\Gamma_{\mathrm{D}}}=g$ on a fixed grid $\left.\mathcal{T}_{h}\right)$ in the case where $\eta_{\mathrm{R}, K}=\eta_{\Gamma_{\mathrm{N}}, K}=0$, i.e. when $f$ is piecewise constant and $\Gamma_{\mathrm{N}}=\emptyset$ or when $u$ is piecewise constant on $\mathcal{E}_{h}^{\mathrm{N}}$. In the general case, if $f$ and $u$ have sufficient regularity, the asymptotic exactness and asymptotic robustness still hold true (this time asymptotic also with respect to $h \rightarrow 0$ ). Although we only use the Oswald interpolate of $\tilde{p}_{h}$ instead of evaluating or approximating the infimum in (5.1), the numerical experiments in the next section show that the estimators of Section 4.1 are almost asymptotically exact, and this also in the convection-diffusion-reaction case. More comments on this point are given in [24,23]. Finally, local efficiency (semi-robust in the sense that the local efficiency constant only depends on local variations in the coefficients and becomes optimal as the local Péclet number gets sufficiently small) of our estimators is proved in these references. 

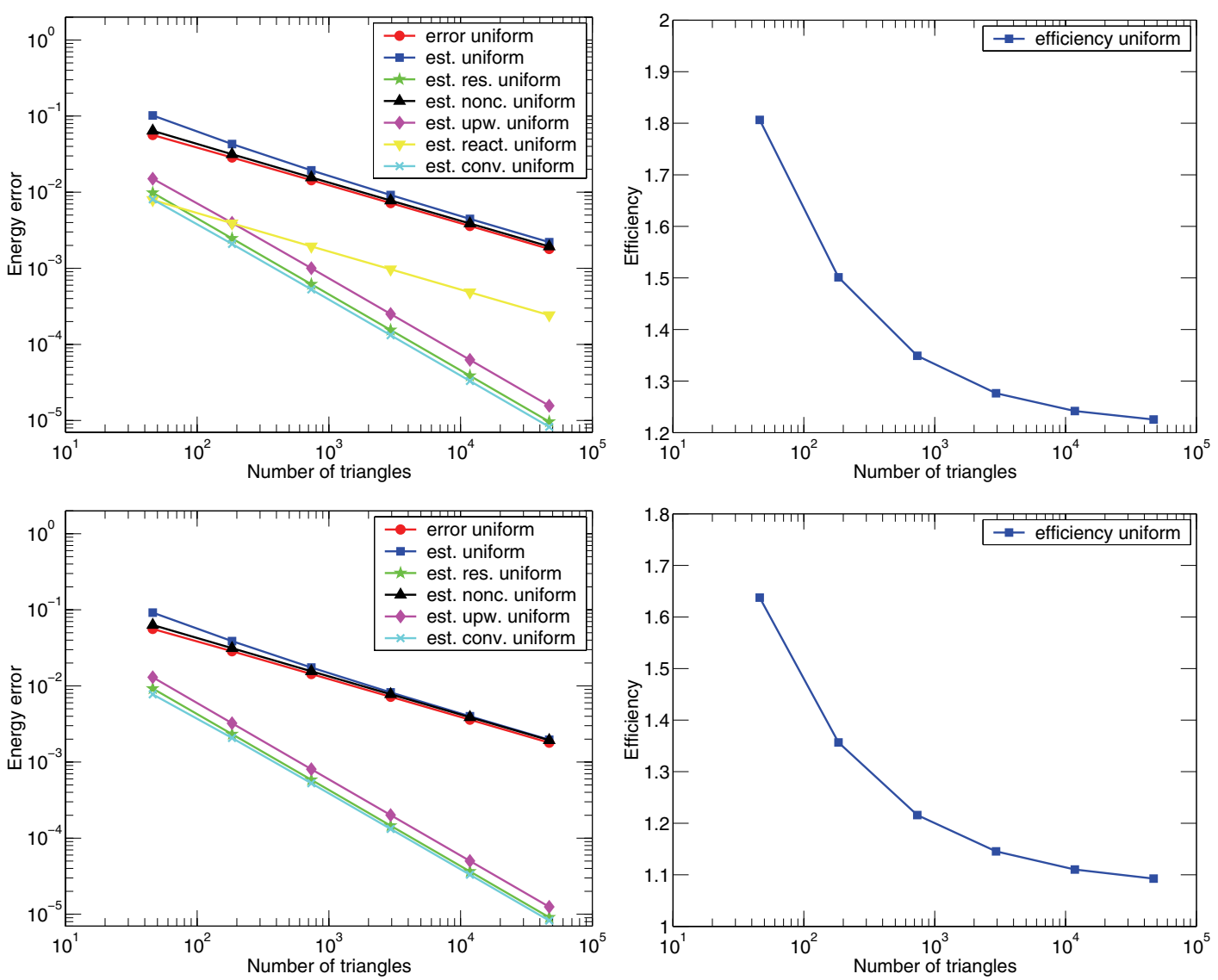

FiguRE 1. Estimated and actual error and the different estimators (left) and overall efficiency (right) against the number of elements, $\varepsilon=1, a=0.5$. Finite volumes (top), mixed finite elements (bottom)

\subsection{Relations between finite volumes and mixed finite elements}

It is shown in [22] that in the lowest-order mixed finite element schemes, there exist local flux expressions. These schemes are thus equivalent to particular finite volume schemes, can be implemented with only one unknown $\left(p_{K}\right)$ per element, and their computational cost can be considerably reduced. This result complements the previously know fact that the finite volume method and the mixed finite element one only differ by numerical integration (see e.g. $[2,3]$ ). The proximity of the two schemes is also confirmed numerically in the next section.

\section{NumericAl EXPERIMENTS}

We illustrate here the behavior of our estimates on a model convection-diffusion-reaction problem. We use the finite volume scheme (3.1)-(3.6) with the local Péclet upstream weighting described in [23, Remark 3.1], which we extend to strictly Delaunay triangular meshes, cf. [10, Example 9.1]. For mixed finite elements, we use a scheme representing a smooth transition from the upwind-weighted scheme $(3.8 \mathrm{a})-(3.8 \mathrm{~b})$ to the centered scheme (3.7a)-(3.7b), see [24, Section 5.5]. These conceptions are important since they imply a second-order discretization of the convection term in the diffusion-dominated case.

We put $\Omega=(0,1) \times(0,1), \mathbf{w}=(0,1)$, and $r=1$ in (1.1a) and consider three cases with $\mathbf{S}=\varepsilon I d$ and $\varepsilon$ equal to, respectively, $1,10^{-2}$, and $10^{-4}$. The right-hand side term $f$, Neumann boundary conditions on 

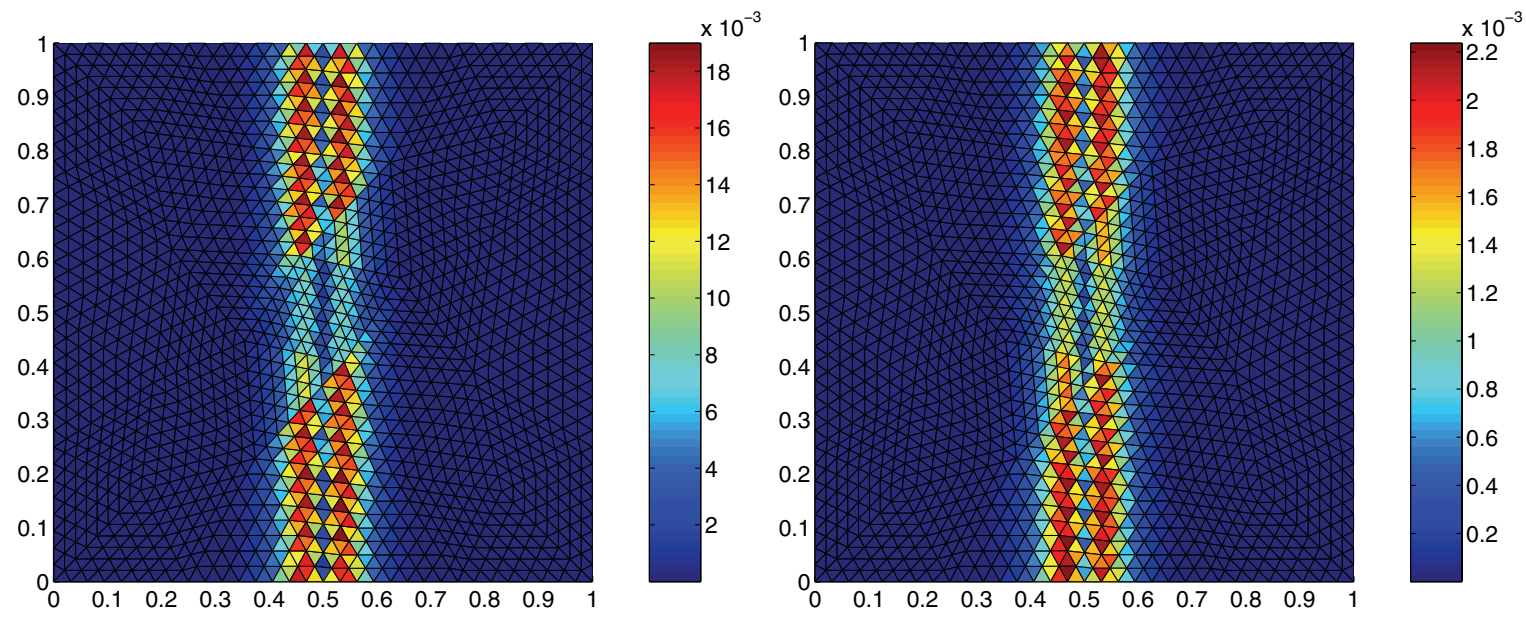

Figure 2. Estimated (left) and actual (right) error distribution, $\varepsilon=10^{-2}, a=0.05$, finite volumes

the upper side, and Dirichlet boundary conditions elsewhere are chosen so that the solution was $p(x, y)=$ $0.5(1-\tanh ((0.5-x) / a)$. This solution is in fact one-dimensional and possesses an internal layer of width $a$ which we set, respectively, equal to $0.5,0.05$, and 0.02 . We start the computations from an unstructured grid of $\Omega$ consisting of 46 triangles and refine it either uniformly (up to 5 refinements) or adaptively.

For $\varepsilon=1$ and $a=0.5$ (diffusion-dominated regime), estimated and actual errors in the energy norm (2.5) and the different estimators for both finite volumes and mixed finite elements on uniformly refined grids are reported in the left part of Figure 1. First of all, the two schemes give very close approximate solutions. Next, since $r$ is a constant, it follows from (3.9b) that the reaction quadrature estimator is zero in the mixed finite element method, and this is the only remarkable difference between the estimators of the two schemes $\left(\mu_{K}=1\right.$ in the finite volume case). Since the residual, convection, and upwinding estimators all represent higher-order terms (the Neumann boundary one is zero), in both cases the only important estimator is the nonconformity one. Overall efficiency, giving the ratio of the estimated $\left|\||\cdot|\|_{\Omega}\right.$-error to the actual $\||\cdot|\|_{\Omega}$-error, is then plotted in the right part of Figure 1. This quantity simply expresses how many times we have overestimated the actual error - recall that there are no undetermined multiplicative constants in our estimates. These plots extend the asymptotic exactness of our estimators discussed in Section 5.2. Finally, the estimators reproduced very precisely the distribution of the error in this case.

For $\varepsilon=10^{-2}$ and $a=0.05$ (convection-dominated regime on coarse meshes and diffusion-dominated regime with progressive refinement), still the distribution of the error in the finite volume case is predicted very well, cf. Figure 2. Note in particular the correct localization of the error away from the center of the shock, as well as the sensitivity of our estimator to the shape of the elements. The results for mixed finite elements on uniformly refined grids are almost identical. Next, the estimated and actual errors for $\varepsilon=10^{-2}$ and $a=0.05$ and $\varepsilon=10^{-4}$ and $a=0.02$ are plotted against the number of elements in uniformly/adaptively refined meshes in Figure 3. One can see that for both schemes, we can substantially reduce the number of unknowns necessary to attain the prescribed precision using the derived a posteriori error estimates and adaptively refined grids. Concerning efficiency, it is similar for both methods and close to two for finest grids in the first case, whereas in the second one, not even the elements in the refined shock region start to leave the convection-dominated regime, so that the efficiency if far from optimal values.

Let us conclude by some additional comments. First of all, the above results show that there are no essential differences in the precision between the considered finite volume and mixed finite element schemes, confirming thus the remarks of Section 5.3. However, recall that the mixed finite element schemes (3.7a)-(3.7b) or (3.8a)-(3.8b) can be defined on any simplicial mesh and easily take into account general data (in particular 

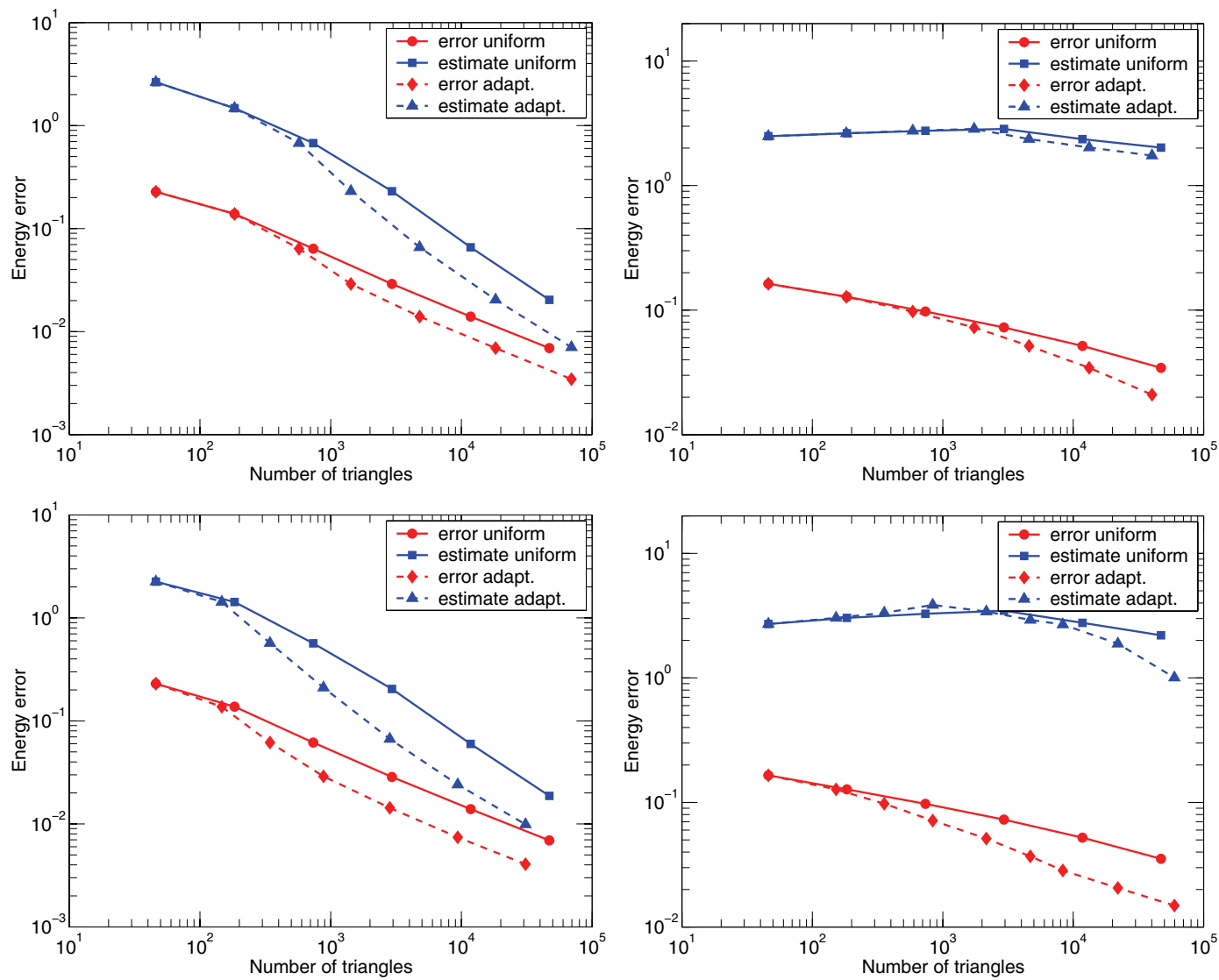

FIGURE 3. Estimated and actual error against the number of elements in uniformly/adaptively refined meshes for $\varepsilon=10^{-2}, a=0.05$ (left) and $\varepsilon=10^{-4}, a=0.02$ (right). Finite volumes (top), mixed finite elements (bottom)

inhomogeneous and anisotropic diffusion-dispersion tensors). With the techniques from [22], one can also significantly reduce their computational cost. Considering the finite volume scheme (3.1)-(3.6), it has first of all quite severe mesh constrains. In particular, in the above simulations, an important effort had to be made in order to keep the adaptively refined mesh uniformly strictly Delaunay, since the usual "longest edge" refinement or its variants cannot be used, see [23] for details. Also, its quadrature-like behavior while taking into account the data is well represented in our simulations by the nonzero reaction estimator. However, this scheme is easier to implement and the arising linear systems are still less expensive to solve. Most importantly, concerning a posteriori error estimation and adaptivity, we come to basically same results. Finally, similar conclusions can be drawn from the numerical results on a model problem with strongly inhomogeneous diffusion-dispersion tensor, which in particular confirm the excellent efficiency and robustness of our estimators in this case, see [24,23].

\section{REFERENCES}

[1] Aavatsmark, I., Barkve, T., BøE, Ø., And Mannseth, T. Discretization on unstructured grids for inhomogeneous, anisotropic media. I. Derivation of the methods. SIAM J. Sci. Comput. 19, 5 (1998), 1700-1716.

[2] Arbogast, T., Dawson, C. N., Keenan, P. T., Wheeler, M. F., and Yotov, I. Enhanced cell-centered finite differences for elliptic equations on general geometry. SIAM J. Sci. Comput. 19, 2 (1998), 404-425.

[3] Baranger, J., Maître, J.-F., And Oudin, F. Connection between finite volume and mixed finite element methods. RAIRO Modél. Math. Anal. Numér. 30, 4 (1996), 445-465. 
[4] Bebendorf, M. A note on the Poincaré inequality for convex domains. Z. Anal. Anwendungen 22, 4 (2003), $751-756$.

[5] Bernardi, C., And Verfürth, R. Adaptive finite element methods for elliptic equations with non-smooth coefficients. Numer. Math. 85, 4 (2000), 579-608.

[6] Carstensen, C. A posteriori error estimate for the mixed finite element method. Math. Comp. 66, 218 (1997), 465-476.

[7] Coudière, Y., Vila, J.-P., And Villedieu, P. Convergence rate of a finite volume scheme for a two-dimensional convectiondiffusion problem. M2AN Math. Model. Numer. Anal. 33, 3 (1999), 493-516.

[8] Dawson, C. Analysis of an upwind-mixed finite element method for nonlinear contaminant transport equations. SIAM J. Numer. Anal. 35, 5 (1998), 1709-1724.

[9] Douglas, JR., J., And Roberts, J. E. Global estimates for mixed methods for second order elliptic equations. Math. Comp. 44, 169 (1985), 39-52.

[10] Eymard, R., Gallouët, T., And Herbin, R. Finite volume methods. In Handbook of Numerical Analysis, Vol. VII. NorthHolland, Amsterdam, 2000, pp. 713-1020.

[11] Eymard, R., Gallouët, T., and Herbin, R. Finite volume approximation of elliptic problems and convergence of an approximate gradient. Appl. Numer. Math. 37, 1-2 (2001), 31-53.

[12] Eymard, R., Gallouët, T., and Herbin, R. A cell-centred finite-volume approximation for anisotropic diffusion operators on unstructured meshes in any space dimension. IMA J. Numer. Anal. 26, 2 (2006), 326-353.

[13] Nicaise, S. A posteriori error estimations of some cell-centered finite volume methods. SIAM J. Numer. Anal. 43, 4 (2005), $1481-1503$.

[14] NiCAISE, S. A posteriori error estimations of some cell centered finite volume methods for diffusion-convection-reaction problems. SIAM J. Numer. Anal. 44, 3 (2006), 949-978.

[15] Ohlberger, M. A posteriori error estimate for finite volume approximations to singularly perturbed nonlinear convectiondiffusion equations. Numer. Math. 87, 4 (2001), 737-761.

[16] Payne, L. E., And Weinberger, H. F. An optimal Poincaré inequality for convex domains. Arch. Rational Mech. Anal. 5 (1960), 286-292 (1960).

[17] Petzoldt, M. A posteriori error estimators for elliptic equations with discontinuous coefficients. Adv. Comput. Math. 16, 1 (2002), 47-75.

[18] Raviart, P.-A., And Thomas, J.-M. A mixed finite element method for 2nd order elliptic problems. In Mathematical aspects of finite element methods (Proc. Conf., Consiglio Naz. delle Ricerche (C.N.R.), Rome, 1975). Springer, Berlin, 1977, pp. 292-315. Lecture Notes in Math., Vol. 606.

[19] Verfürth, R. A review of a posteriori error estimation and adaptive mesh-refinement techniques. Teubner-Wiley, Stuttgart, 1996.

[20] Verfürth, R. A posteriori error estimators for convection-diffusion equations. Numer. Math. 80, 4 (1998), 641-663.

[21] Vohralík, M. On the discrete Poincaré-Friedrichs inequalities for nonconforming approximations of the Sobolev space $H^{1}$. Numer. Funct. Anal. Optim. 26, 7-8 (2005), 925-952.

[22] Vohralík, M. Equivalence between lowest-order mixed finite element and multi-point finite volume methods on simplicial meshes. M2AN Math. Model. Numer. Anal. 40, 2 (2006), 367-391.

[23] Vohralík, M. Residual flux-based a posteriori error estimates for finite volume discretizations of inhomogeneous, anisotropic, and convection-dominated problems. submitted (2006).

[24] Vohralík, M. A posteriori error estimates for lowest-order mixed finite element discretizations of convection-diffusion-reaction equations. SIAM J. Numer. Anal., accepted for publication (2007). 